Tabelle II.

\begin{tabular}{|c|c|c|c|c|c|c|c|}
\hline $\begin{array}{l}\text { Bestandteile } \\
\text { (g in } 100 \mathrm{ccm} \text { ) }\end{array}$ & $\begin{array}{l}\text { Nr. } 1 \\
\text { Weißse } \\
\text { Trie } \\
\text { Wein }\end{array}$ & $\begin{array}{l}\text { Nr. } 2 \\
\text { nd rote } \\
\text { rer } \\
\text { ipfel }\end{array}$ & $\begin{array}{c}\text { Nr. } 3 \\
\text { Rote } \\
\text { Trierer } \\
\text { Woin- } \\
\text { apfel }\end{array}$ & $\begin{array}{c}\text { Nr. } 4 \\
\text { Weice } \\
\text { Trierer } \\
\text { Wein- } \\
\text { apfel }\end{array}$ & $\begin{array}{c}\text { Nr. 5. } \\
\text { Ge- } \\
\text { mischter } \\
\text { Obstwein; } \\
\text { Apfel } \\
\text { und } \\
\text { Girnen } \\
\end{array}$ & $\begin{array}{l}\text { Nr. } 6 \\
\text { Birnen } \\
\text { wein }\end{array}$ & $\begin{array}{c}\text { Nr. } 7 \\
\text { Ge- } \\
\text { misehter } \\
\text { Birnen- } \\
\text { woin }\end{array}$ \\
\hline Spezifisches Gewicht. . & 1,0027 & 1,0026 & 1,0031 & 1,0026 & 1,0143 & 1,0076 & 1,0139 \\
\hline Alkohol . . . . . . . . & 3,99 & 3,99 & 4,05 & 4,29 & 3,87 & 4,53 & 4,11 \\
\hline Gesamt Extrakt . . . . . . & 2,73 & 2,69 & 2,75 & 2,60 & 5,51 & 3,98 & 5,53 \\
\hline Unvergorener Zucker.... & 0,11 & 0,14 & 0,08 & 0,20 & 2,09 & 0,13 & 1,84 \\
\hline Zuckerfreier Extrakt. . . . & 2,72 & 2,65 & 2,75 & 2,50 & 3,52 & 3,95 & 3,79 \\
\hline Gesamt-Säure (Apfelsänre) $\therefore$ & 0,63 & 0,64 & 0,77 & 0,97 & 0,52 & 1,07 & 0,76 \\
\hline Flüchtige Säure & 0,07 & 0,07 & 0,07 & 0,06 & 0,04 & 0,09 & 0,05 \\
\hline Nichtflüchtige Säure (Apfelsäure) & 0,55 & 0,56 & 0,69 & 0,90 & 0,48 & 0,97 & 0,70 \\
\hline Weinsäure . . . . . . & 0 & 0 & 0 & 0 & 0 & 0 & 0 \\
\hline Milchsäure , . . . . . & 0,96 & 0,96 & 0,90 & 0,56 & 0,39 & 0,09 & 0,25 \\
\hline $\begin{array}{l}\text { Zuckerfreier Extrakt minus freie } \\
\text { Säure } \cdot \cdot \cdot \cdot \cdot \cdot \cdot \cdot \cdot \cdot \cdot \cdot \cdot \\
\end{array}$ & 2,17 & 2,09 & 2,06 & 1,60 & 3,04 & 2,98 & 3,09 \\
\hline $\begin{array}{l}\text { Zuckerfreier Extrakt minus nicht- } \\
\text { flüchtige Säure . } . \cdot \cdot \cdot \cdot\end{array}$ & 2,09 & 2,01 & 1,98 & 1,53 & 3,00 & 2,88 & 3,03 \\
\hline Glycerin . . . . . & 0,41 & 0,39 & 0,40 & 0,37 & 0,26 & 0,34 & 0,29 \\
\hline $\begin{array}{l}\text { Verhältnis von Alkohol zu Gly- } \\
\text { cerin }=100:\end{array}$ & 10,3 & 9,8 & 9,9 & 8,6 & 6,7 & 7,5 & 7,0 \\
\hline Mineralstoffe $\cdot \cdot \cdots \cdot \cdot \cdot \cdot \cdot \cdot$ & 0,310 & 0,302 & 0,324 & 0,259 & 0,327 & 0,351 & 0,375 \\
\hline Gesamt-Alkalität $=\operatorname{com}$ N.-Lauge & 4,3 & 4,2 & 3,6 & 2,8 & 4,0 & 3,8 & 4,5 \\
\hline $\begin{array}{l}\text { Wasserlösliche Alkalität }=\mathrm{ccm} \\
\text { N.-Lauge } \cdot \cdot \cdot \cdot \cdot \cdot \cdot \cdot \cdot\end{array}$ & 3,4 & 2,4 & 3,1 & 2,3 & 2,5 & 2,1 & 3,2 \\
\hline
\end{tabular}

\title{
Über den Nachweis geringer Mengen von Formaldehyd
} und von einigen Formaldehydverbindungen mit Fuchsinsehwefligsalzsänre.

Von

\section{Heinrich Fineke in Köln.}

Der einwandfreie Nachweis von Formaldehyd ist, wenn es sich um geringe Mengen handelt, nicht immer leicht zu führen. Die Schwierigkeiten, die hierbei trotz der großen Zahl von Formaldehydreaktionen bestehen, werden besonders klar, wenn man das Schrifttum durchsieht, das sich auf den Formaldehydnachweis in grünen 
Pflanzen bezieht. Seitdem Baeyer seine bekannte Assimilationshypothese aufgestellt hat, nach der Formaldehyd das wesentlichste Zwischenprodukt bei dem Aufbau der Kohlenhydrate aus der Kohlensäure der Luft sein soll, ist immer wieder der Versuch gemacht worden, Formaldehyd in grünen Pflanzen nachzuweisen. Angeblich ist der Versuch wiederholt erfolgreich gewesen, doch hat sich nachher jeder vermeintliche Nachweis als unsicher oder irrig herausgestellt 1 ).

Versuche, die ich vor kurzem über den Formaldehydgehalt der Pflanzen und über die Möglichkeit des Nachweises geringer Formaldehydmengen darin anstellte, ergaben folgendes ${ }^{2}$ ):

1. Die Formaldehydreaktion mit Fuchsinschwefligsalzsäure nach Grosse-Bohle ${ }^{3}$ ) eignet sich zum Nachweis geringer Formaldehydmengen bei Gegenwart anderer organischer Stoffe, auch in Pflanzensäften. Die Reaktion gestattet eine annähernde colorimetrische Bestimmung.

2. In den ausgepreßten und filtrierten $S$ äften belichteter grüner Blätter verschiedener Pflanzen war Formaldehyd mittels Fuchsinschwefligsalzsäure n icht aufzufinden. Künstlicher Formaldehydzusatz war in einer Verdünnung $1: 100000$ bis $1: 200000$ zu erkennen ${ }^{4}$ ).

3. In den Destillaten von Blättern verschiedener Pflanzen war Formaldehyd auch dann nicht aufzufinden, wenn den Blättern vorher geringe Mengen Formaldehyd zugesetzt waren. Dies hat seinen Grund darin, daf frischer wie erhitzter Brei grüner und nichtgrüner Pflanzen schon bei gewöhnlicher Temperatur und besonders beim Erhitzen, selbst bei Gegenwart von Phos. phorsäure, Formaldehyd zerstören oder binden. Auch lebenden Blättern einverleibter Formaldehyd findet sich nach kurzer Zeit nicht mehr darin.

Diese Ergebnisse waren Veranlassung, weitere Versuche über Formaldehydnachweis anzustellen. Da das Verfahren mittels Fuchsinschwefligsalzsäure anscheinend weniger als die anderen Nachweisverfahren durch gleichzeitige Anwesenheit fremder Stoffe beeinflubt wird, verdient es weitere Anwendung, als es bisher gefunden hat. Ich hatte - weniger für nahrungsmittelchemische als für biochemische Zwecke - den Wunsch, die Empfindlichkeit der Reaktion, die in farblosen Lösungen bis etwa $1: 500000$, in gefärbten Lösungen weniger beträgt, zu erhöhen. Abänderung der Reagensiösung führte aber nicht zum Ziel, doch gelang es, die Empfindlichkeit in gefärbten Lösungen durch Entfernung der störenden Stoffe zu vergrößern.

Für den Nachweis von Formaldehyd in Nahrungsmitteln hat vor allem die Tatsache Interesse, daß Formaldehyd unter bestimmten Verhältnissen zerstört wird, wie dies bei "der Destillation mit Formaldehyd versetzten Pflanzenbreies beobachtet war. Diese Möglichkeit ist sowohl beim Nachweis wie bei der quantitativen Bestimmung von Formaldehyd zu beachten, wenn andere organische Stoffe zugegen sind. Die Frage, wie weit ein solehes Verschwinden von Formaldehyd bei Nahrungsmitteln

1) Vergl. hierzu Bokorny, Chem.-Ztg. 1909, 33, 1141; Curtius und Franzen, Sitzungsber. d. Heidelberger Akad., Math.-Naturw. Klasse 1912, 7. Abhandl.; Fincke, Biochem. Zeitschr. 1913, 52, 214.

2) Biochem. Zeitschr. 1913, 52, 214.

3) Diese Zeitschrift, 1907 14, 89.

${ }^{4}$ Nachträglich wurden noch einige Pflanzensäfte unter Bedingungen geprïft, unter denen eine Formaldehydverdünnung 1:500000 nach eben erkennbar war; auch hierbei wurde Formaldehyd nicht gefunden. 
in Betracht kommt, konnte zwar nicht erschöpfend behandelt werden, doch ergaben sich verschiedene Anhaltspunkte

Endlich wurde festgestellt, wie sich Hexamethylentetramin und Formaldehydnatriumbisulfit der Fuchsinschwefligsalzsäure gegenüber verhalten.

\section{Reagens und Reaktion.}

1. Die fuchsinschweflige $\mathrm{S}$ äure bereitet man am besten ${ }^{1}$ ), indem man $1 \mathrm{~g}$ reines essigsaures oder salzsaures Rosanilin in etwa $500 \mathrm{ccm}$ Wasser lost und dieser Lösung eine wässerige Lösung von $25 \mathrm{~g}$ krystallisiertem Natriumsulfit sowie $15 \mathrm{ccm}$ Salzsäure (spez. Gewicht 1,124) zusetzt; dann füllt man zu 11 auf. Die sich langsam entfärbende Flüssigkeit ist nach einigen Stunden gebrauchsfertig und bei Aufbewahrung in verschlossenen Gefäßen lange haltbar.

Die Reaktion wird nach Grosse-Boble in folgender Weise ausgeführt: $10 \mathrm{ecm}$ der zu prüfenden Flüssigkeit werden mit (1 oder) $2 \mathrm{ccm}$ Salzsäure (spez. Gewicht 1,124) gemischt; dann fügt man $1 \mathrm{ccm}$ fuchsinschweflige Säure hinzu. Bei Anwesenheit von Formaldehyd tritt je nach dessen Menge in einigen Minuten bis einigen Stunden, spätestens in 12 Stunden, eine langs am zunehmende, haltbare, blau- bis rotviolette Färbung ein. Stoffe, die in saurer Lösung Formaldehyd abspalten, wie Hexamethylentetramin und Methylal, geben die Reaktion natürlich ebenfalls.

Diese Reaktion hat sich unter mannigfachen Bedingungen bewährt; vor allem macht sie in vielen Fällen eine Destillation unnötig, da sie meistens im Untersuchungsobjekt oder dessen Auszug ausführbar ist.

Für das Gelingen der Reaktion ist es erforderlich, sich genau an die Vorschrift zu halten; die zu untersuchende Flüssigkeit ist zuerst mit der Salzsäure zu mischen, ehe man das Fuchsinreagens zugibt. Auch die angegebenen Mengenverhältnisse sind einzuhalten, da sie sich bei der Nachprüfung als zweckmäßig ergeben haben. Der Salzsäurezusatz kann, wenn Spuren von Formaldebyd nachgewiesen werden sollen und größere Mengen anderer Aldehyde nicht vorhanden sind, auf $1 \mathrm{ccm}$ zu $10 \mathrm{ccm}$ Flüssigkeit ermäßig werden; bei der doppelten Salzsäuremenge $(2 \mathrm{ccm})$ treten Störungen durch andere Aldebyde weniger leicht ein, doch erfolgt dann der Reaktionseintritt etwas langsamer. Die im Reagens enthaltene schweflige Säure befindet sich bereits im Überschuß; ein nicht allzu großer weiterer Überschuß stört nicht.

Von der allgemeinen Aldehydreaktion mit Fuchsinschwefligsäure unterscheidet sich die Formaldehydreaktion durch die Gegenwart von viel freier Säure. Am besten eignet sich Salzsäure; Denigés ${ }^{2}$ ) führt die Reaktion unter Verwendung von Schwefelsäure aus. Bei der angegebenen Reaktion stören andere Aldebyde in normaler Weise rorkommenden Mengen nicht. Auch die verschiedenartigsten anderen Stoffe zeigten sich ohne Einfluß.

Es wurde versucht, das Rosanilin bei der Reaktion durch ähnliche Farbstoffe zu ersetzen. Hochmethylierte Fuchsine sowie Säurefuchsin (Rosanilintrisulfosaures Natrium) und Säureviolett (Dimethylrosanilintrisulfosaures Natrium) waren nicht brauchbar. Die beiden letztgenannten Farbstoffe werden zwar durch Natriumsulfit annähernd entfärbt, doch kehrt die Färbung - wenn auch nicht in voller Stärke - auf Salz-

1) Persönliche Mitteilung von Dr. Grosse-Bohle.

2) Diese Zeitscbrift 1912, 28, 229. 
säurezusatz zurück. Weder die neutrale, durch Natriumbisulfit entfärbte, noch die saure bisulfithaltige, mit Wasser bis zu schwacher Färbung verdünnte Säurefuchsinund Säureviolettlösung wird durch Formaldehyd erheblich verändert.

Ferner wurden folgende Fuchsinhomologe miteinander verglichen, die von den Höchster Farbwerken freundlichst zur Verfügung gestellt waren:

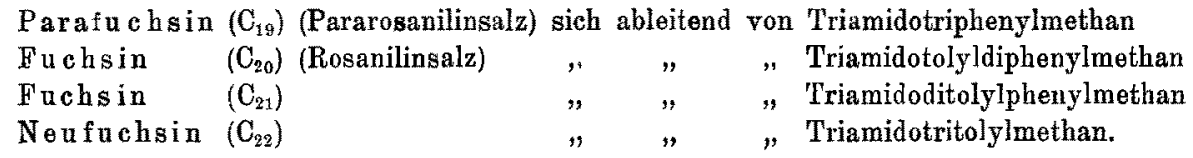

Diese Farbstoffe werden durch Natriumsulfit entfärbt; die Entfärbung bleibt auch bei Salzsäurezusatz bestehen. Die Lösungen von Parafuchsin und Neufuchsin wurden allerdings nicht völlig farblos, sondern hellbräunlich; am hellsten wurde die Lösung von Fuchsin $\left(\mathrm{C}_{20}\right)$.

Die vier Fuchsinhomologen geben die Formaldehydreaktion ziemlich gleichmäBig; nur mit dem Unterschiede, daß bei gleichen Formaldehydmengen die entstehende Farbenstärke sich rom Parafuchsin zum Neufuchsin, also mit steigendem Molekulargewicht, abschwächt. Es gilt dies sowohl bei der Anwendung gleicher Gewichtsmengen der Farbstoffe, wie bei der Anwendung aequimolekularer Mengen. Der Unterschied in der Farbenstärke der Reaktion ist jedoch nicht sehr erheblich. Der Farbenton der Reaktion mit Parafuchsin ist bei geringen Formaldehydmengen mehr rötlich, derjenige mit den Fuchsinen $\left(\mathrm{C}_{20}-\mathrm{C}_{22}\right)$ mehr bläulich. Da die bläuliche Färbung der Reaktion mit Fuchsin $\left(\mathrm{C}_{20}\right)$ ebenso gut zu erkennen ist wie die etwas stärkere rötlichere Färbung der Parafuchsinreaktion, so bietet die Anwendung von Parafuchsin keinen Vorteil. Es empfiehlt sich Fuchsin $\left(\mathrm{C}_{20}\right)$ beizubehalten.

Die Konstitution des bei der Formaldehydreaktion mit Fuchsinschwefligsalzsäure entstehenden Farbstoffes ist anscheinend noch nicht aufgeklärt, obgleich die Herstellung dieses Farbstoffes Gegenstand eines D.R.P. ist. Der wesentliche Bestandteil des Fuchsinschwefligsäurereagens, die Rosanilinchlorhydrat-Leukosulfonsäure $\mathrm{NH}_{2}, \mathrm{C}_{3} \mathrm{H}_{4}$

$\mathrm{HCl} . \mathrm{NH}_{2}, \mathrm{C}_{6} \mathrm{H}_{3}\left(\mathrm{CH}_{3}\right)>\mathrm{C}-\mathrm{SO}_{3} \mathrm{H} \cdot \mathrm{H}_{2} \mathrm{O}$ ist von $\mathrm{Hantzsch}$ und $\left.\mathrm{OBwald}{ }^{1}\right)$ in $\mathrm{NH}_{2} \cdot \mathrm{C}_{6} \mathrm{H}_{4}$

fester Form dargestellt. Zweifellos wird die Färbung nicht durch den Farbstoff Fuchsin, sondern durch ein gefärbtes Kondensationsprodukt bewirkt. folgendes:

Versuche, die Bedingungen der Bildung desselben klarzulegen, ergaben

Andere Aldehyde können eine Färbung verursachen, wenn sie in übergroßen, praktisch kaum vorkommenden Mengen anwesend sind. Geringe Mengen anderer Aldehyde geben Färbung, wenn die Salzsäuremenge verringert wird. Nur bei hoher Säurekonzentration ist die Reaktion für Formaldehyd charakteristisch. Die Reaktion tritt um so leichter ein, je größer die vorhandene Fuchsinmenge ist; alsdann stören aber auch andere Aldehyde eher, so daß die Fuchsinmenge in der angegebenen mittleren Menge anzuwenden ist.

Durch Natriumsulfit allein entfärbte Fuchsinlösung gibt die Reaktion nicht; sie tritt ein, wenn man alsbald Säure zusetzt. Zur Farbstoffbildung ist somit Säure erforderlich.

1) Ber. Deutsch. Chem. Gesellsch. 1900, 38, 311. 
Versetut man Fuchsinlösung mit konzentrierter Salzsäure $(1: 10)$, so erfolgt Entfärbung. Die so entfärbte Fuchsinlösung wird durch Formaldehyd nicht verändert, sondern erst dann, wenn man noch Sulfit oder schweflige Säure zufügt. Die Farbstoffbildung ist also an die Gegenwart von schwefliger Säure gebunden.

Formaldehydschweflige Säure reagiert als solche nicht mit Fuchsinschwefligsalzsäure. Gibt man zu den auf Formaldehyd zu prüfenden Lösungen zuerst Fuchsinschwefligsalzsäurereagens, so wird Formaldehyd allmählich gebunden und bei nachträglichem Salzsäurezusatz nur teilweise zur Reaktion gebracht. Man kann die allmäbliche Bindung von Formaldehyd durch Natriumbisulfit deutlich verfolgen, wenn man auf $1 \mathrm{ccm}$ einer $0,4 \%$-igen Formaldehydlösung $1 \mathrm{ccm}$ einer $5 \%$-igen Natrium. bisulfitlösung verschieden lange (z. B. 0, 1, 5, 10, 20 Minuten) einwirken läßt, dann auf $10 \mathrm{ccm}$ verdünnt und je $1 \mathrm{ccm}$ Salzsäure und Fuchsinschwefligsäure zufügt.

\section{Nachweis von Formaldehyd.}

Meist führt man den Formaldehydnachweis im Destillate der Nahrungsmittel. Bei dem Fuchsinschwefligsalzsäureverfahren ist Destillation in den meisten Fällen entbehrlich, Erforderlich ist sie, wenn die Reaktion in der Substanz selbst oder in ihrem Auszuge wegen nicht entfernbarer starker Färbung nicht ausführbar ist. Destillation ist auch dann zweckmäfig, wenn auf allergeringste Mengen Formaldehyd (1:500000 bis 1:1000000) gefahndet werden soll, da bei der Destillation, auch bei der Wasserdampfdestillation größerer Flüssigkeitsmengen, eine Anreicherung des Formaldehyds im ersten Destillate erzielt wird. Doch ist hierfür Bedingung, daß bei der Destillation Formaldehyd nicht gebunden wird. Findet dagegen beim Erhitzen der zu prüfenden Substanz eine chemische Bindung von Formaldehyd statt, die auch durch zweckentsprechende Zusätze nicht verhindert wird, so kann man geringe Mengen Formaldehyd sicherer in der Substanz oder in ihrem wässerigen Auszuge direkt nachweisen. Selbst in schwach gefärbten Lösungen wird bei einer Formaldehydkonzentration 1:100000 die Reaktion innerhalb einiger Stunden sichtbar. Die direkte Prüfung der Substanz oder ihres Auszuges wird daher in den meisten Fällen ohne weiteres ausreichend sein.

Ist bei der Destillation Verlust an Formaldehyd wahrscheinlich, dann ist die direkte Prüfung die richtigere; im einzelnen Falle ist nötigenfalls durch einen Gegenversuch zu prüfen, ob dies zutrifft. Völliges Verschwinden des Formaldehyds bei der Destillation kommt wohl nur in Frage, wenn nur geringe Mengen desselben vorhanden sind; aber auch ein teilweises Verschwinden ist nicht unbeachtet zu lassen, selbst nicht in den Fällen, in denen eine genaue Mengenbestimmung nicht stattfinden soll; denn es ist stets wünschenswert, aus der Stärke der Reaktion einen annähernden Schluß auf die Menge zu ziehen. Bei quantitativen Formaldehydbestimmungen ist der etwaige Formaldehydverlust bei der Destillation natürlich besonders zu beachten; in ungünstigen Fällen wird die colorimetrische Bestimmung im Untersuchungsobjekt oder dessen Auszug der einzige gangbare Weg sein.

Die Fuchsinschwefligsalzsäure-Reaktion kann ohne weiteres angestellt werden, wenn eine farblose Lösung vorliegt. Alkalische Lösungen sind zunächst zu neutralisieren. Ferner kann die Reaktion ohne weiteres in Milch ausgeführt werden; gerade für den Formaldehydnachweis in Milch wurde die Reaktion ursprünglich von GrosseBohle in erster Linie angegeben. Die Empfindlichkeitsgrenze in Milch fand ich etwa bei einer Formaldehydkonzentration 1:400000, wenn man auf $10 \mathrm{ccm}$ Milch 
$1 \mathrm{ccm}$ Salzsäure anwendet. Dies stimmt mit der Angabe Grosse-Bohle's überein, daß ein Tropfen Formalin (40\%-ig) noch in 5 bis 101 Milch (d. i. 1:250000 bis $1: 500000)$ nachweisbar sei.

Stark gefärbte Lösungen, wie Rotweine, Fruchtsäfte, Pflanzensäfte, Hackfleischauszüge, entfärbt man vorher mit Tierkoble. Die zu benutzende Tierkohle prüft man auf Formaldehydgehalt und reinigt sie nötigenfalls durch Auswaschen mit heißem Wasser. Die Entfärbung vollzieht sich am besten, indem man die zu prüfende Lösung zunächst mit der für die Reaktion notwendigen Salzsäure $(1-2 \mathrm{ccm}$ Salzsäure vom spez. Gew. 1,124 auf $10 \mathrm{ccm}$ Flüssigkeit) versetzt, dann mit Tierkohle schüttelt und filtriert. Erfolgt eine Entfärbung auch dann schlecht, so führt kurzes Erwärmen der salzsauren Lösung mit der Tierkohle meist zum Ziel, wobei allerdings in einzelnen Fällen Formaldehydverlust eintreten könnte. Durch die Behandlung mit Tierkohle werden geringe Formaldehydmengen nicht beeinflußt. Statt der Behandlung mit Tierkohle kann man zuweilen auch Fällen der Lösung mit konzentrierter Mer. curiacetatlösung ( $\boldsymbol{~}$. B. bei Rotwein und Fruchtsäften) oder Mercurichloridlösung (bei Fleischauszügen) anwenden. Bei gewöhnlicher Temperatur wird auch durch sie Formaldehyd in starker Verdünnung nicht angegriffen.

Durch die Entfärbung gelingt es fast stets, die Empfindlichkeit der Reaktion in Flüssigkeiten auf 1:300000 bis 1:500000 zu steigern. Gerade solche geringe Mengen entgehen dem Nachweise bei der Destillation in manchen Fällen.

Die Abnahme des Formaldehydgehaltes in Nahrungsmitteln und anderen organischen Stoffen kann zwei Ursachen haben: Chemische Umwandlung oder Bindung des Formaldehyds oder Zerstörung durch die Tätigkeit von Kleinlebewesen.

Die rein chemischen Ursachen des Verschwindens von Formaldehyd können mannigfach sein; er reagiert mit den verschiedenartigsten Stoffen, doch ist bei der Untersuchung solcher Reaktionen, wie zum Beispiel der Einwirkung von. Formaldebyd auf Eiweißstoffe und deren Abbauprodukte, im allgemeinen mit höheren Formaldehydkonzentrationen gearbeitet worden, als sie hier Berücksichtigung finden sollen. In sehr verdünnter Lösung sind einzelne Reaktionen sehr abgeschwächt. Daß aber auch sehr verdünnter Formaldehyd leicht in Reaktion gezogen werden kann, zeigt das Verhalten von Blätterbrei. Die Größe der formaldehydbindenden Kraft desselben ergibt sich daraus, daß zum Beispiel ein Formaldehydzusatz 1:10000 zu Phabarberblätterbrei nach 1-stündigem Erhitzen auf $100^{\circ}$ nicht mehr nachweisbar war. Welcher Stoff in diesem Falle den Formaldehyd bindet, ist nicht aufgeklärt. Harn, auch Harnstofflösungen, binden Formaldehyd ebenfalls leicht. Die chemischen Verbindungen, die Formaldehyd eingeht, bilden zum Teil Formaldehyd leicht zurück, zum Beispiel Hexamethylentetramin und Methylal bei Finwirkung von Säuren, formaldehydschweflige Säure beim Erwärmen mit Alkalicarbonat. Aus anderen Formaldehydkondensationsprodukten ist Formaldehyd nicht oder nur schwer wieder freizumachen. Blätterbrei, der Formaldehyd aufgenommen hat, gibt auch dann keine Formaldehydreaktion, wenn man ihn unter Zusatz von Salzsäure erhitzt hat, vielmehr bindet Blätterbrei Formaldehyd auch bei Salzsäuregegenwart. Allzu starke Mittel zur Spaltung der Formaldehydkondensationsprodukte darf man bei der Untersuchung: organischer Objekte nicht anwenden, um nicht Gefahr zu laufen, daß Formaldehyd durch Zersetzung anderer organischer Stoffe entsteht. Verschiedene Untersuchungsobjekte verhalten sich in verschiedener Richtung verschieden; man wird daher das 
Verhalten im einzelnen Falle durch einige Versuche aufklären müssen, wenn es sich darum handelt, den Beweis für Gegenwart oder Abwesenheit ganz geringer Formaldehydmengen zu erbringen.

Die chemische Bindung von Formaldehyd durch Mileh ist gering. Erhitzt man Milch, die mit Formaldehyd 1:25000 bis 1:100000 versetzt ist, eine halbe Stunde im Wasserbade, so geht die Formaldehydkonzentration etwa auf die Hälfte herunter. Erhitzt man jedoch unter Pbosphorsäurezusatz (1:10), so findet keine merkliche Formaldehydabnahme statt. Man kann somit durch Destillation unter Phosphorsäurezusatz in Milch vorhandenen Formaldehyd verlustlos ins Destillat überführen. Das gleiche gilt für Butter, Margarine und andere Fette. Bei Fleisch tritt dagegen unter gleichen Verhältnissen ein geringer Formaldehydverlust ein. In Fruchtsirupen, Honig und anderen wesentlich aus $\mathrm{Zucker}$ bestehenden Stoffen findet eine merkbare chemische Bindung geringer Formaldehydmengen nicht statt.

Auch die biologische Bindung von Formaldehyd ist zu beachten. Versetzt man Milch, frisches Fleisch, Käse, verdünnte Zuckerlösungen mit geringen Formaldehydmengen, so findet eine Formaldebydabnahmedurch die Tätigkeit von Kleinlebewesen statt.

Drei Proben ungekochter Milch wurden mit Formaldehyd 1:10000, 1:50000 und 1:100000 versetzt und bei gewöhnlicher Temperatur stehen gelassen. Nach 5 Tagen war in der ersten Probe die Konzentration auf etwa 1:25000 und in der zweiten auf etwa 1:300000 gesunken; die dritte Probe ließ Formaldehyd nicht mehr nachweisen. Bei gekochter Milch war die Abnahme geringer. Ähnlich verhielten sich Fleisch und Käse. In einer verdünnten Honiglösung ging der Formaldehydgehalt in 6 Tagen von 1:10000 auf 1:50000 zurück.

Hieraus ergibt sich zunächst, daß man zum Konservieren von Stoffen, die gute Nährböden sind, nicht zu geringe Formaldehydmengen anwenden darf. Ferner darf man die Untersuchung solcher Stoffe auf etwaigen Formaldehydgehalt nicht hinausschieben. Endlich kann man aus dem Nichtauffinden von Formaldehyd in Stoffen, die starke Kleinlebewesenentwickelung zeigen, nicht den Schlub ziehen, daß sie einen Formaldehydzusatz nicht erhalten baben.

Wendet man die Fuchsinschwefligsalzsäure zur colorimetrischen Formaldehydbestimmung an, so nimmt man ähnlich zusammengesetzte Vergleichslösungen. Die zu prüfende Lösung und die Vergleichsflüssigkeit müssen - mit Ausnahme von Milch - beide völlig farblos sein oder - wenn dies nicht zu erreichen ist - den gleichen Farbenton besitzen. Zur Bestimmung in Milch stellt man die Vergleichslösung ebenfalls mit Milch her. Wegen der Schärfe der Reaktion ist colorimetrischer Vergleich nur bei starker Formaldehydverdünnung möglich. Die zu vergleichenden Proben sind zu gleicher Zeit anzusetzen.

\section{Nachweis von Hexamethylentetramin.}

Die Formaldehydschwefligsalzsäure-Reaktion tritt auch mit Hexamethylentetramin ein, da dieses durch Salzsäure gespalten wird. Diese Spaltung ist bei gewöhnlicher Temperatur nicht in kurzer Zeit vollständig, so daß Hexamethylentetramin langsamer und weniger stark reagiert, wie die entsprechende Formaldehydmenge. Nach 15-stündigem Stehen der Reaktion ist eine Hexamethylenkonzentration 1:200000 in farblosen Lösungen immerhin erkennbar. Eine völlige Spaltung des Hexamethylentetramins erzielt man, indem man 10 Teile der Lösung mit 1 oder 2 Teilen Salz- 
säure (spez. Gew. 1,124) 10 Minuten in siedendem Wasserbade erhitzt; die Reaktion tritt dann ebenso leicht wie mit der entsprechenden Formaldehydmenge ein. Bei gefärbten Flüssigkeiten verbindet man die Spaltung mit der Entfärbung durch Tierkohle. Auch bei der Destillation aus phosphorsaurer Lösung wird Hexamethylentetramin völlig gespalten.

Es ist zu berücksichtigen, daß 1 Molekül Hexamethylentetramin (140,2) 6 Molekülen Formaldehyd $(180,1)$ entspricht; 100 Teile Hexamethylentetramin geben also 128,4 Teile Formaldehyd.

\title{
Nachweis von Formaldehydschwefligsäure.
}

Formaldehydschwefligsäure bildet sich in freiem Zustande oder als Salz, wenn Formaldehyd oder Hexamethylentetramin mit Schwefligsäure oder Bisulfiten zusammentreffen; sie entsteht somit beim Zusatz von Formaldehyd oder Hexamethylentetramin zu geschwefelten Weinen.

Formaldehydschwefligsäure reagiert nur in sehr geringem Maße mit Fuchsinschwefligsalzsäure, nur insoweit als aus ihr Formaldehyd durch Salzsäure abgespalten wird. In der Kälte findet dies nur in geringem Grade statt. Selbst Erhitzen mit Salzsäure in der bei Hexamethylentetramin angegebenen Weise oder Destillation unter Phosphorsäurezusatz genügen nicht, um den gesamten Formaldehyd abzuspalten. Erst durch Destillation mit größerem Natriumcarbonatüberschuß gelingt es, Formaldehyd völlig freizumachen. Eine solche Behandlung ist bei Nahrungsmitteln wegen der Möglichkeit, daß sich damn auch durch Zersetzung anderer Stoffe Formaldehyd bildet, meist nicht anwendbar. Man kann daher bei ihnen im allgemeinen Formaldehyd in Form der Schwefligsäureverbindung nicht leicht quantitativ bestimmen oder mit gleicher Schärfe nachweisen wie freien oder als Hexamethylentetramin vorhandenen Formaldehyd.

\section{Versuche zur Theorie der Entmanganung von Grundwasser. Von}

\author{
Dr. J. Tillmans und 0. Heublein.
}

Mitteilungaus der Chemisch-hygienischen Abteilung (Abteilungsvorsteher: Dr. J. Tillmans) des Städtischen Hygienischen Instituts in Frankfurta. M. (Direktor: Professor Dr. M. Neisser).

Seit der großen Breslauer Wasserkalamität vom Jahre 1906 schenkt man allgemein dem Vorkommen von Mangansalzen im Trinkwasser die größte Beachtung. Zwar hat schon Proskauer im Jahre 1894 auf das Vorkommen von Mangansalzen im Wasser hingewiesen ${ }^{1}$, indessen ist dieser Hinweis verhältnismäßig wenig beachtet geblieben, bis die Katastrophe in Breslau die allgemeine Aufmerksamkeit auf die Mangansalze lenkte.

1) Vergl. den offiziellen Bericht über die XVIII. Hauptversammlung des Preuf. Medizinalbeamtenvereins zu Berlin 1901, s. 58 . 